\title{
Tau Pathology and Parietal White Matter Lesions Have Independent but Synergistic Effects on Early Development of Alzheimer's Disease
}

\author{
Joakim Hertze $\mathrm{a}^{\mathrm{a}} \mathrm{b}$ Sebastian Palmqvist ${ }^{\mathrm{a}, \mathrm{c}}$ Lennart Minthon ${ }^{\mathrm{a}, \mathrm{b}}$ \\ Oskar Hansson ${ }^{\mathrm{a}, \mathrm{b}}$ \\ ${ }^{a}$ Clinical Memory Research Unit, Department of Clinical Sciences, Lund University, and \\ ${ }^{b}$ Memory Clinic, Skåne University Hospital, Malmö, and ' ${ }^{\mathrm{C}}$ Department of Neurology, \\ Skåne University Hospital, Lund, Sweden
}

Key Words

Alzheimer's disease · Dementia · Cerebrospinal fluid · White matter lesions · Follow-up studies

\section{Abstract}

Background: White matter lesions (WMLs) are a common finding in patients with dementia. This study investigates the relationship between WMLs, hyperphosphorylated tau ( $P$-tau) in cerebrospinal fluid (CSF) and apolipoprotein E (APOE) $\varepsilon 4$ genotype in prodromal Alzheimer's disease (AD). Methods: Baseline levels of tau, P-tau and $\beta$-amyloid 1-42 in CSF, the presence of WMLs in the brain, and the APOE genotype were ascertained in 159 patients with mild cognitive impairment (MCI) and 38 cognitively healthy controls. Results: After 5.7 years, 58 patients had developed AD. In this group, patients with normal levels of CSF P-tau had higher levels of WMLs in the parietal regions than those with pathological P-tau levels $(p<0.05)$. Also, patients without APOE $\varepsilon 4$ alleles had more WMLs in the parietal lobes than those with at least one allele $(p<0.05)$. MCI patients with pathological P-tau levels and parietal WMLs showed a greater risk of developing $A D$ than those with just one of the two pathological parameters. Conclusions: We suggest that WMLs in parietal lobes and tau pathology likely have independent but synergistic effects on the reduction of the cognitive reserve capacity of the brain. In patients with a more low-grade AD pathology, WMLs in the parietal lobes might increase the risk of developing dementia. 


\section{Introduction}

Dementia is a condition that causes great personal tragedy as well as one which has a major economic impact on society as a whole $[1,2]$. The number of people affected is likely to double every 20 years [3], making better diagnostic methods and treatments a growing concern. While the latter decades of life in general are characterized by cognitive decline, measurable at a population level, individual trajectories are very diverse. Some people progress to dementia, while others barely show any change at all [4].

Alzheimer's disease (AD), the most common cause of dementia [5], is a heterogeneous condition, most likely consisting of a family of related, but different, aetiologies. The clinical course of AD varies from a slow deterioration, spanning decades, to aggressive forms, annihilating cognition within a few years. Neuropathological hallmarks of AD are senile plaques containing $\beta$-amyloid $(A \beta)$ and neurofibrillary tangles containing hyperphosphorylated tau (P-tau). Notable risk factors for $\mathrm{AD}$ are age, carrying the $\varepsilon 4$ allele of the apolipoprotein $\mathrm{E}$ (APOE) gene and factors related to the reserve capacity of the brain, such as education and vascular risk factors [6].

The most common early symptom of $\mathrm{AD}$ is a reduction of episodic memory. At this stage patients usually meet the criteria of 'mild cognitive impairment' (MCI). This is a heterogeneous syndrome, wherein 30-60\% have prodromal AD while the rest have a stable form of memory impairment or are in the early stages of another type of dementia, such as vascular dementia (VaD) [7]. So far there is no reliable way to predict the fate for individual patients with MCI.

White matter lesions (WMLs), which can be detected using both magnetic resonance imaging (MRI) and computer tomography (CT), are common in older populations. A few studies have shown that WMLs in people with MCI increase the risk of future dementia $[8,9]$, but others contradict this finding $[10,11]$. While previous studies have investigated the role of WMLs in full-blown dementia due to AD $[12,13]$, less is known about how these changes affect patients with milder memory complaints.

In this study we investigate the relationship between $\mathrm{AD}$ pathology, represented by cerebrospinal fluid (CSF) P-tau and $\beta$-amyloid 1-42 (A $\beta 42)$, the presence of APOE $\varepsilon 4$ alleles, and the prevalence of WMLs in patients who progress from MCI to AD.

\section{Material and Methods}

\section{Patients with AD, MCI and Cognitively Healthy Controls}

This study was performed at the Memory Clinic of Skåne University Hospital in Malmö. CSF samples were obtained from 38 cognitively healthy controls and 166 individuals seeking healthcare for milder memory complaints who were diagnosed as suffering from MCI. At the clinical baseline visit, physicians with a special interest in cognitive disorders performed a thorough physical, neurological and psychiatric examination, as well as a clinical interview focusing on cognitive symptoms and performance in the activities of daily living. Cognitive tests, analysis of APOE genotype, and CT or MRI of the brain were performed. Patients with a clinical syndrome of MCI were followed clinically for an average period of 5.7 years (range: 3.0-9.6). Of the 166 patients with MCI, 7 had to be excluded from further study due to missing CT scans.

Patients with MCI at baseline had to fulfil the criteria advocated by Petersen [7], including: (1) memory complaint, preferably corroborated by an informant; (2) objective memory impairment adjusted for age and education, as judged by the physician; (3) preservation of general cognitive functioning, as determined by the clinician's judgment based on a struc- 
tured interview with the patient and a Mini-Mental Status Examination (MMSE) score greater than or equal to 24; (4) zero or minimal impairment of daily life activities, and (5) not fulfilling the DSM-III-R criteria of dementia [14]. Patients with other causes of cognitive impairment, such as subdural haematoma, brain tumour, CNS infection, schizophrenia, major depressive episode and current alcohol abuse were not included.

MCI patients who received a diagnosis of AD during clinical follow-up were required to meet the DSM-III-R criteria for dementia [14] and the criteria for probable AD defined by NINCDS-ADRDA [15]. Subjects who during follow-up were diagnosed as having VaD fulfilled the DSM-III-R criteria for dementia and the requirements for probable VaD by NINDS-AIREN [16]. VaD of the subcortical type was diagnosed according to the recommendations by Erkinjuntti et al. [17]. The consensus criteria by McKeith et al. [18] were used when diagnosing dementia with Lewy bodies.

The control population consisted of healthy elderly volunteers who were recruited from Malmö, Sweden. Inclusion criteria were (1) absence of memory complaints or any other cognitive symptoms, (2) preservation of general cognitive functioning, and (3) no active neurological or psychiatric disease. The clinical diagnosis for each patient was reviewed by a consensus group of three medical doctors (J.H., L.M. and O.H.) with a special interest in cognitive disorders. The Regional Ethics Committee in Lund, Sweden, approved the study and the patients and/or their relatives gave their informed consent (for research).

\section{Analysis of Baseline CSF}

CSF was collected in polypropylene tubes, stored at $-80^{\circ} \mathrm{C}$ and analysed after the clinical follow-up of the study was completed. The procedure followed The Alzheimer's Association flow chart for LP and CSF sample processing [19]. The levels of A 342 , tau and tau phosphorylated at Thr181 (P-tau) were determined using xMAP technology as previously described [20].

\section{Assessment of WMLs}

All WMLs were assessed by S.P. with a protocol developed by Wahlund et al. [21] (AgeRelated White Matter Changes scale, ARWMC). This scale was developed for rating both MRI and CT images, with high agreement between the modalities [21]. Twenty-two subjects were examined with MRI using a 1.5-tesla scanner from Siemens, and the other subjects were examined with noncontrast CT using different CT scanners. The flair sequences of the MRI images were used for rating the WMLs. S.P. was blinded to the follow-up diagnoses. The WMLs were assessed separately in the left and right frontal regions, the basal ganglia and the occipital-parietal regions - hereafter referred to as the parietal region since most WMLs were located there. Temporal lesions were not rated since this is a very rare location for WMLs and therefore unsuitable for statistical analysis [22]. The lesions were graded from 0 to 3 points: $0=$ no lesions or lesions $<5 \mathrm{~mm}, 1=$ presence of lesion $\geq 5 \mathrm{~mm}, 2=$ lesions beginning to aggregate, and $3=$ confluent lesions involving almost the entire region. Lesions (infarcts) in the basal ganglia were rated slightly different: 0 points $=$ no lesions, $1=$ one lesion of at least $2 \mathrm{~mm}, 2$ = more than one lesion, and 3 = confluent lesions.

The mean intrarater reliability based on 20 randomized patients was $\kappa=0.86$, indicating excellent agreement according to Altman [23]. Interrater reliability with a co-developer of the ARWMC scale and exact definitions of the assessed regions have been described previously [24].

\section{Statistical Analyses}

The statistical analyses were made with IBM SPSS for Macintosh, version 19.0.0 (IBM Corp., Somers, N.Y., USA). To compare demographic and CSF baseline data between groups, a nonparametric Kruskal-Wallis test was performed followed by a Mann-Whitney U test for 
Hertze et al.: Tau Pathology and Parietal White Matter Lesions Have Independent but Synergistic Effects on Early Development of Alzheimer's Disease

Table 1. Demograpic data, with CSF levels of P-tau and WML assessments

\begin{tabular}{lcccc}
\hline & Controls & Stable MCI & Prodromal AD & MCI-other \\
\hline Females/males & $27 / 11$ & $39 / 36$ & $40 / 18^{\mathrm{e}, \mathrm{d}}$ & $10 / 16^{\mathrm{a}}$ \\
Age, years & $77 \pm 8$ & $69 \pm 7^{\mathrm{b}}$ & $76 \pm 7^{\mathrm{c}, \mathrm{e}}$ & $73 \pm 7^{\mathrm{c}}$ \\
Carrier of APOE $\varepsilon 4$ & $10(26)$ & $33(44)$ & $44(76)^{\mathrm{b}, \mathrm{d}, \mathrm{e}}$ & $12(46)$ \\
MMSE score at baseline & $28.3 \pm 1.8$ & $28.2 \pm 1.3$ & $26.1 \pm 1.6^{\mathrm{b}, \mathrm{d}, \mathrm{e}}$ & $27.0 \pm 1.9^{\mathrm{a}, \mathrm{c}}$ \\
CSF P-tau, ng/ml & $31 \pm 17$ & $30 \pm 15$ & $52 \pm 22^{\mathrm{b}, \mathrm{d}, \mathrm{f}}$ & $30 \pm 12$ \\
CSF tau, ng/ml & $91 \pm 49$ & $80 \pm 48$ & $141 \pm 68^{\mathrm{b}, \mathrm{d}, \mathrm{f}}$ & $80 \pm 38$ \\
CSF Aß, ng/ml & $265 \pm 74$ & $252 \pm 61$ & $154 \pm 55^{\mathrm{b}, \mathrm{d}, \mathrm{f}}$ & $226 \pm 77$ \\
Left frontal WMLs & $0.34 \pm 0.63$ & $0.36 \pm 0.63$ & $0.62 \pm 0.77^{\mathrm{c}}$ & $0.96 \pm 1.1^{\mathrm{a}, \mathrm{c}}$ \\
Right frontal WMLs & $0.29 \pm 0.52$ & $0.39 \pm 0.72$ & $0.59 \pm 0.75$ & $0.96 \pm 1.1^{\mathrm{a}, \mathrm{c}}$ \\
Left parietal WMLs & $0.21 \pm 0.62$ & $0.31 \pm 0.66$ & $0.53 \pm 0.75^{\mathrm{a}, \mathrm{c}}$ & $0.88 \pm 1.2^{\mathrm{a}, \mathrm{c}}$ \\
Right parietal WMLs & $0.32 \pm 0.62$ & $0.29 \pm 0.63$ & $0.59 \pm 0.82^{\mathrm{c}}$ & $0.96 \pm 1.1^{\mathrm{a}, \mathrm{d}}$ \\
Sum frontal WMLs & $0.63 \pm 1.2$ & $0.75 \pm 1.3$ & $1.2 \pm 1.5$ & $1.9 \pm 2.1^{\mathrm{a}, \mathrm{c}}$ \\
Sum parietal WMLs & $0.53 \pm 1.2$ & $0.6 \pm 1.3$ & $1.1 \pm 1.5^{\mathrm{a}, \mathrm{c}}$ & $1.8 \pm 2.3^{\mathrm{a}, \mathrm{c}}$ \\
Total sum frontoparietal WMLs & $1.2 \pm 2.0$ & $1.3 \pm 2.4$ & $2.3 \pm 2.7^{\mathrm{a}, \mathrm{c}}$ & $3.8 \pm 4.0^{\mathrm{a}, \mathrm{c}}$ \\
Left basal ganglia infarcts & $0.08 \pm 0.27$ & $0.16 \pm 0.37$ & $0.17 \pm 0.48^{\mathrm{e}}$ & $0.58 \pm 0.81^{\mathrm{b}, \mathrm{c}}$ \\
Right basal ganglia infarcts & $0.05 \pm 0.23$ & $0.18 \pm 0.51$ & $0.26 \pm 0.58$ & $0.19 \pm 0.40$ \\
Sum basal ganglia infarcts & $0.13 \pm 0.34$ & $0.34 \pm 0.73$ & $0.45 \pm 0.88^{\mathrm{e}}$ & $0.77 \pm 0.95^{\mathrm{a}, \mathrm{c}}$ \\
Total sum WMLs (including BG) & $1.3 \pm 2.0$ & $1.6 \pm 2.7$ & $2.8 \pm 3.0^{\mathrm{a}, \mathrm{d}}$ & $4.5 \pm 4.4^{\mathrm{a}, \mathrm{d}}$ \\
\hline
\end{tabular}

Data are means \pm SD or number $(\%)$. Stable MCI $=$ MCI patients with stable cognitive functions during a follow-up period of 3.0-9.6 years; prodromal $\mathrm{AD}=\mathrm{MCI}$ patients who developed AD during follow-up; $\mathrm{WML}=$ white matter lesions in subcortical brain matter; $\mathrm{BG}=$ basal ganglia. ${ }^{\mathrm{a}} \mathrm{p}<0.05,{ }^{\mathrm{b}} \mathrm{p}<0.005$ vs. controls; ${ }^{\mathrm{c}} \mathrm{p}<0.05$, ${ }^{\mathrm{d}} \mathrm{p}<0.005$ stable MCI; ${ }^{\mathrm{e}} \mathrm{p}<0.05$, ${ }^{\mathrm{f}} \mathrm{p}<0.005$ vs. MCI-other. Correction for multiple analyses has not been performed.

continuous variables. Pearson's $\chi^{2}$ test was used for dichotomous variables. The Spearman correlation coefficient was used for bivariate correlation analyses.

We added up WMLs in the frontal lobes, parietal lobes and basal ganglia, respectively, disregarding very slight periventricular white matter changes $(<5 \mathrm{~mm})$ in the frontal lobes since these are a very common finding in healthy elderly and should, according to the ARWMC scale, not be considered a WML [21]. We considered P-tau greater than $51 \mathrm{ng} / \mathrm{ml}$ and A $\beta 42$ less than $209 \mathrm{ng} / \mathrm{l}$ to be pathological, according to cutoffs established in previous work [20]. To compare the presence of WMLs between individuals with pathological and nonpathological CSF levels of P-tau, as well as between individuals with and without at least one APOE $\varepsilon 4$, a Mann-Whitney test was performed for each brain region. A Kaplan-Meier estimate, together with Cox regression, adjusted for age and gender, were used for investigating the probability of conversion from MCI to AD in four groups.

\section{Results}

We studied the remaining 159 patients with MCI at baseline, as well as 38 cognitively healthy controls who all had undergone successful CSF analysis of P-tau and a CT or MRI of the brain. After an average period of 5.7 years (3.0-9.6), 75 of the patients with MCI (47\%) at baseline were still cognitively stable. Fifty-eight patients (36\%) progressed to dementia due to AD, with or without an addition of vascular pathology. Twenty-six patients (24\%) developed other types of dementia, such as $\operatorname{VaD}(n=17)$, dementia with Lewy bodies $(n=4)$, progressive supranuclear palsy $(n=3)$, semantic dementia $(n=1)$ and normal pressure hydrocephalus $(\mathrm{n}=1)$. 
Hertze et al.: Tau Pathology and Parietal White Matter Lesions Have Independent but Synergistic Effects on Early Development of Alzheimer's Disease

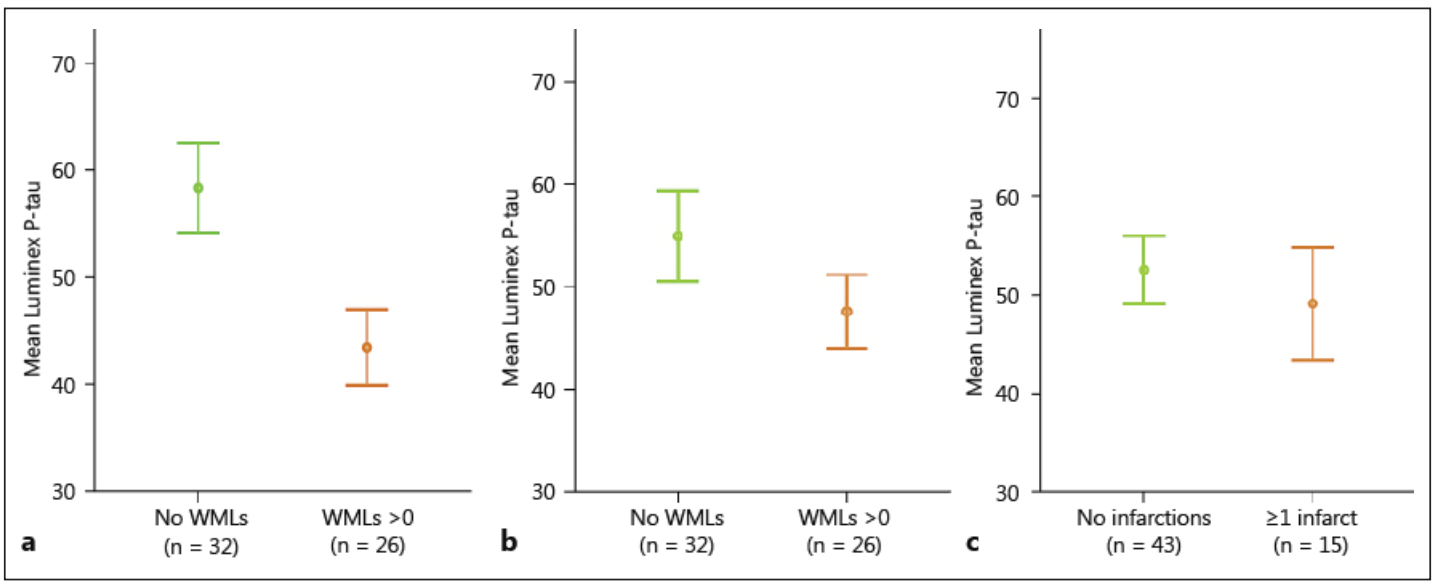

Fig. 1. CSF levels of P-tau in prodromal AD patients with or without WMLs. The P-tau levels are significantly lower in patients with WMLs in the parietal lobes (a), but not in patients with WMLs in the frontal lobes (b) or in patients with infarctions in the basal ganglia $(\mathbf{c})$. Error bars represent $\pm 1 \mathrm{SE}$.

The demographic data, CSF P-tau, CSF tau, CSF A $\beta$ and assessment of WMLs of all included subjects are shown in table 1 . Among the patients with $\mathrm{MCI}$ at baseline who later developed dementia due to AD (prodromal AD), the frequency of APOE $\varepsilon 4$ carriers was significantly higher, the MMSE score at baseline was significantly lower, the CSF levels of tau and P-tau were significantly higher and the CSF levels of A $\beta 42$ were significantly lower when compared to healthy controls, cognitively stable MCI patients or patients with MCI who developed another type of dementia than AD (table 1). Moreover, the patients with prodromal AD exhibited increased levels of WMLs in the parietal areas when compared to controls and cognitively stable MCI (table 1). Patients who developed other types of dementia, mainly VaD, showed significantly more WMLs in all analysed brain regions, as would be expected (table 1).

\section{Pathological WMLs and CSF Biomarkers in Prodromal AD}

In the group with prodromal $\mathrm{AD}$ patients, $45 \%$ had visible white matter changes $(\geq 1$ point on the ARWMC scale) and $46 \%$ had pathological levels of P-tau in CSF (defined as P-tau $>51 \mathrm{ng} / \mathrm{l}[20]$ ), while $90 \%$ had pathological levels of A 342 in CSF (defined as A $\beta 42<209 \mathrm{ng} / \mathrm{l}$ [20]). This means the vast majority of patients with prodromal AD showed A $\beta$ pathology, but only a subset had white matter changes or increased P-tau levels. We therefore studied the relationship between P-tau levels and white matter changes in this patient group.

In the group with prodromal AD, we found the CSF levels of P-tau to be significantly lower in patients who exhibited WMLs in the parietal lobes when compared to patients with prodromal AD who had no WMLs in these brain regions ( $p<0.008$; fig. 1a). No significant differences in CSF P-tau levels were found in prodromal AD cases with WMLs in the frontal lobes or vascular lesions in the basal ganglia (fig. 1b, c). Similarly, prodromal AD patients with normal P-tau levels had more WMLs in the parietal lobes than cases with pathological levels of P-tau ( $p<0.05$; fig. 2a). Also, patients who had no APOE $\varepsilon 4$ alleles showed a larger prevalence of WMLs in the parietal lobes compared to those with one or two APOE $\varepsilon 4$ alleles ( $p<$ 0.05; fig. 2b).

\section{Prediction of AD in Subjects with MCI at Baseline}

Next, we studied the associations between CSF P-tau, WMLs and future development of $\mathrm{AD}$ in the whole cohort with MCI at baseline. Figure 3 shows the Kaplan-Meier estimates of 
Hertze et al.: Tau Pathology and Parietal White Matter Lesions Have Independent but

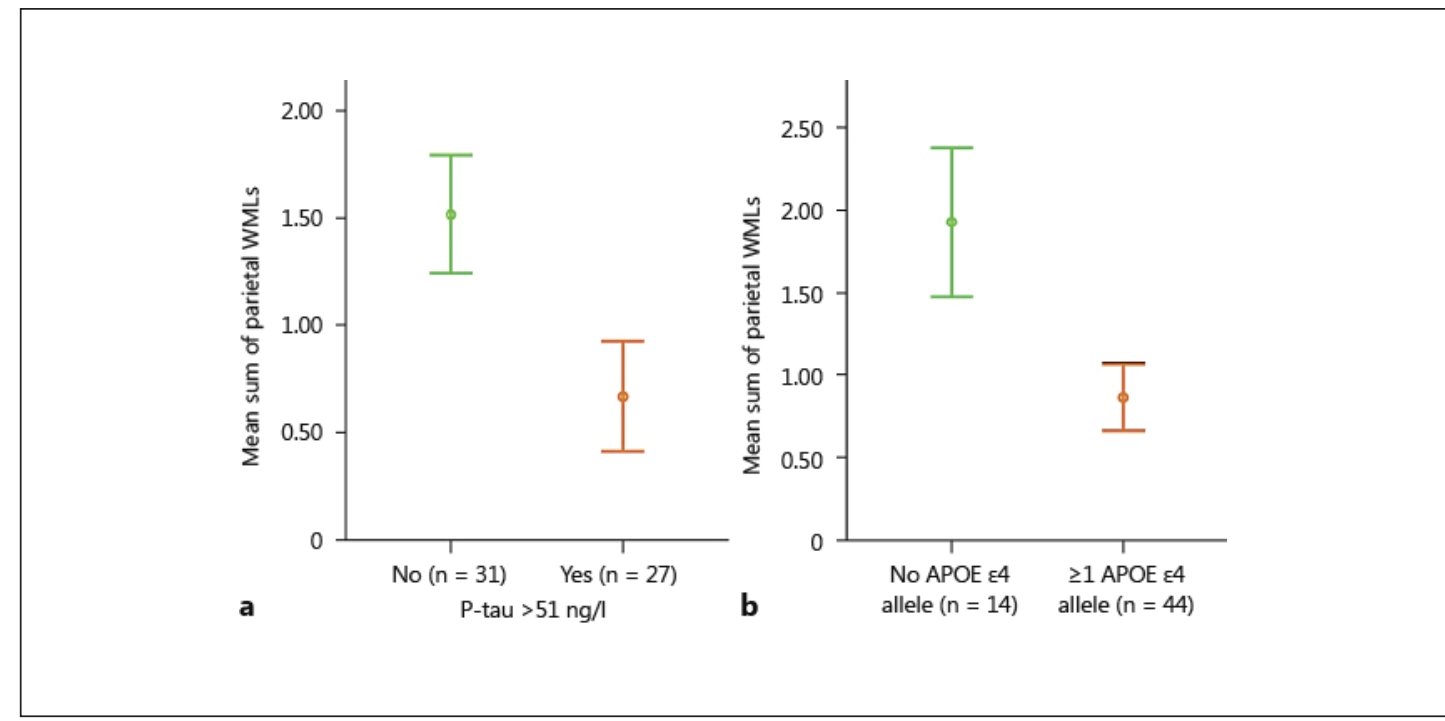

Fig. 2. a There are significantly more WMLs in the parietal lobes, shown as a mean sum, in prodromal AD patients without pathological levels of P-tau, defined as P-tau $>51 \mathrm{ng} / \mathrm{l}$. $\mathbf{b}$ There are also more WMLs in the parietal lobes in patients carrying no APOE $\varepsilon 4$ allele. Error bars represent $\pm 1 \mathrm{SE}$.

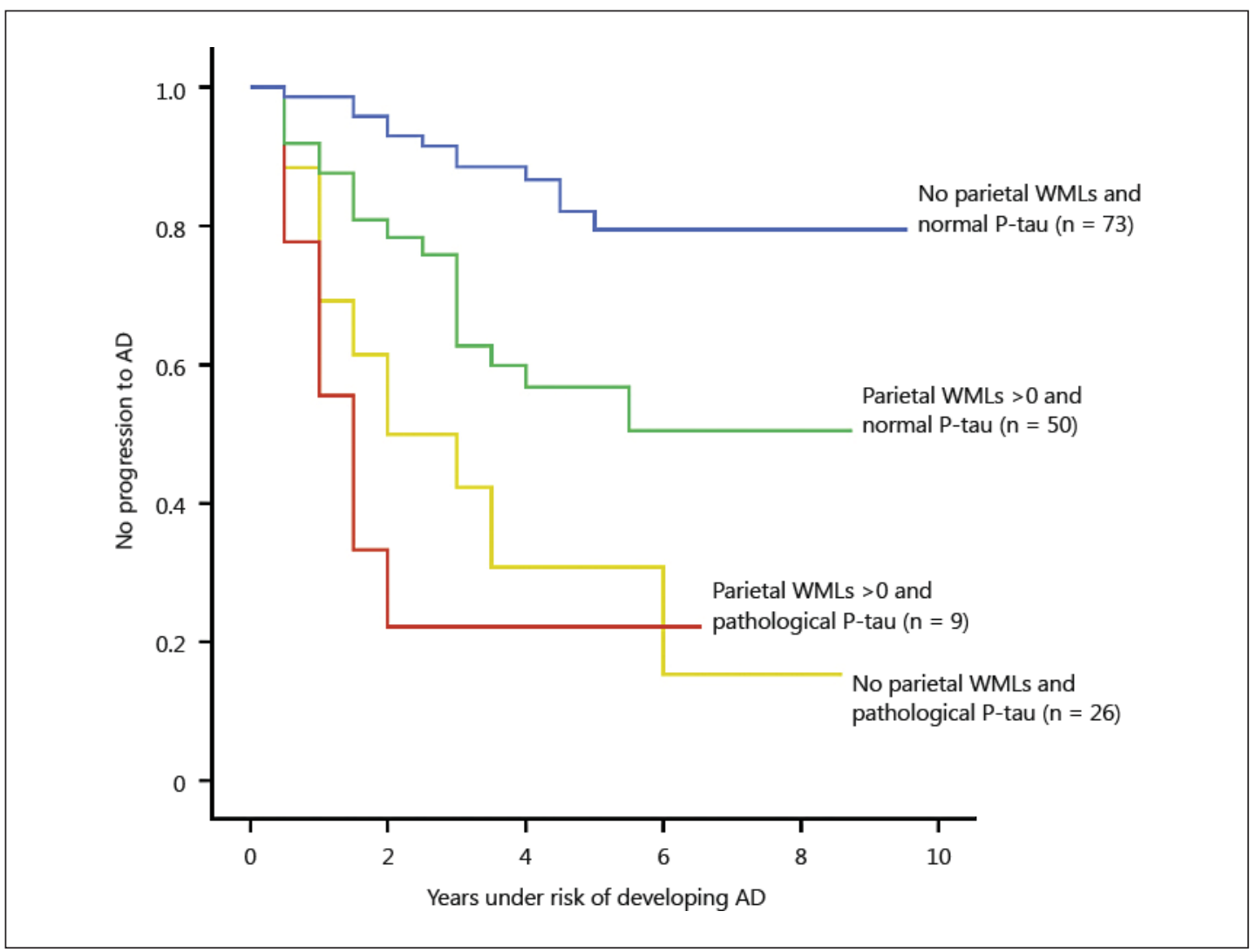

Fig. 3. Kaplan-Meier estimates of the rate of progression to $\mathrm{AD}$ in subjects with $\mathrm{MCI}$ in relation to the levels of CSF P-tau and/or presence of WMLs in the parietal lobes at baseline. MCI cases with both pathological levels of P-tau and/or WMLs in the parietal lobes at baseline progress more rapidly towards AD. One individual was removed from this analysis due to missing data regarding levels of P-tau. 
Table 2. Cox proportional hazards regression models examining the relation between baseline P-tau and WMLs, and the risk of converting to AD in patients with MCI

Unadjusted hazard ratio Adjusted hazard ratio $(95 \% \mathrm{CI})$ $(95 \% \mathrm{CI})^{1}$

Normal P-tau and no parietal WMLs Normal P-tau and presence of parietal WMLs Pathological P-tau and no parietal WMLs Both pathological P-tau and presence of parietal WMLs

$\begin{array}{cl}1.0 \text { (reference) }^{\mathrm{b}} & 1.0 \text { (reference) }^{\mathrm{a}} \\ 3.2(1.5-6.6)^{\mathrm{b}} & 2.3(1.1-4.8)^{\mathrm{a}} \\ 7.0(3.4-14.3)^{\mathrm{c}} & 4.9(2.4-10.2)^{\mathrm{c}} \\ 10.3(4.0-26.4)^{\mathrm{c}} & 6.6(2.5-17.6)^{\mathrm{c}}\end{array}$

All data were collected at baseline. ${ }^{a} \mathrm{p}<0.05 ;{ }^{b} \mathrm{p}=0.002 ;{ }^{\mathrm{c}} \mathrm{p}<0.0001 .{ }^{1}$ Adjusted (if applicable) for the baseline demographic variables: age, sex and APOE $\varepsilon 4$ carrier status.

the probability of conversion to $\mathrm{AD}$ in MCI subjects with either (1) normal P-tau and no parietal WMLs, (2) normal P-tau and the presence of parietal WMLs, (3) pathological P-tau and no parietal WMLs, and (4) both pathological P-tau and the presence of parietal WMLs. MCI patients with both pathological P-tau and parietal WMLs at baseline had a substantially increased risk of future AD, with a hazard ratio of 10.3 (95\% CI: 4.0-26.4, p < 0.001), when compared to those with normal P-tau levels and no parietal WMLs at baseline (table 2; fig. 3).

\section{Discussion}

In our study, patients who seek healthcare with milder memory complaints show less tau pathology when they also exhibit WMLs in the parietal lobes. The added effect of WMLs in the parietal regions seems to lower the threshold for AD-like symptoms to appear.

We used CSF levels of P-tau as a biochemical marker of AD pathology in the brain. Neuropathological studies, as well as PET imaging studies, suggest that altered metabolism of A $\beta$ and the formation of senile plaque occur many years before the onset of symptoms in AD [25]. However, these changes do not correlate well with the severity of the disease [26, 27]. In contrast, the amount of tau-containing neurofibrillary tangles seems to be associated with the stage of cognitive decline throughout the course of the disease [28-31]. While the level of regular tau in CSF is a rather general marker for neuronal damage, the levels of P-tau seem to reflect the phosphorylation state of tau in the brain, and thus the formation of pathological tangles [32].

Earlier work has firmly established the value of CSF biomarkers in predicting conversion from MCI to AD [33-36]. These results were later confirmed in a study by our group [20], and this evidence is generally considered to be convincing. Our study focuses on the synergistic effects between two different biomarkers and suggests the assessment of WMLs to be valuable in predicting the course of disease for at least a subset of AD patients.

Neuropathological studies have shown that a relatively large subgroup of AD patients also have vascular pathology [37, 38]. A few of them have also shown that the addition of vascular lesions to $\mathrm{AD}$ pathology seems to worsen the clinical symptoms of the disease in populations comprised of already demented individuals [12, 13, 39]. Several of them have described findings consistent with our results, i.e. that $\mathrm{AD}$ patients with vascular lesions often exhibit less extensive tau pathology. However, these studies were conducted on already demented individuals who were at different stages of the disease. We studied patients who started out at the same cognitive level, the MCI stage. 
In our material, patients with prodromal AD and normal levels of CSF P-tau exhibited higher levels of WMLs in parietal regions than those with pathological P-tau levels. This indicates that different types of pathology, affecting temporal and parietal brain regions, may result in a clinical presentation of early AD. We also found more WMLs in parietal regions at baseline in individuals not carrying any APOE $\varepsilon 4$ allele, suggesting that in the absence of this risk factor the added burden of WMLs is needed to develop cognitive symptoms. Earlier studies have demonstrated an increase of WMLs in patients with AD as the disease progresses [40, 41]. Our data (fig. 3) takes this one step further, suggesting that individuals with both pathological P-tau and parietal WMLs at baseline exhibit a more rapid progression towards AD.

Maruyama et al. [42] compared CSF tau levels and WMLs between stable MCI patients and $\mathrm{MCI}$ patients who converted to AD. They found the stable MCI group to be characterized by lower levels of tau and a higher grade of periventricular WMLs, while the AD-converted group showed increased levels of CSF tau and low grades of periventricular WMLs. They did not investigate the relationship between tau and WMLs in the group who converted to AD alone. Nevertheless, their data seems to suggest the same as ours: patients with WMLs may develop memory complaints in the absence of tau pathology. While they suggest tau pathology and WMLs comprise two different pathways to a clinical syndrome of cognitive impairment, our study suggests these two pathways may also have synergistic effects on the development of dementia. In addition, Grambaite and Stenset [43], using diffusion tensor imaging, found memory difficulties in MCI patients without tau pathology in CSF to be related with disrupted white matter diffusivity and radial diffusivity.

While an impaired episodic memory is the most common presenting symptom at the stage of MCI, the addition of symptoms arising from a dysfunction in the parietal lobes, such as dysphasia, dyscalculia, dyspraxia and visiospatial problems, usually characterizes the transition to AD dementia. Among our prodromal AD patients, we only found an inverse relationship between CSF P-tau and APOE $\varepsilon 4$ carriership when we looked at WML load in the parietal lobes. Gootjes et al. [44] found something similar when investigating the regional distribution of WMLs in AD and healthy aging. Their findings show significantly more WMLs per brain volume in the parietal and occipital lobes of AD patients. We suggest WMLs in parietal and occipital lobes might lead to cognitive deficiencies in their own right, contributing to a clinical presentation of AD.

Only $45 \%$ of our prodromal AD patients showed pathological levels of CSF P-tau, while $90 \%$ had pathological levels of CSF A 42 . Changes of the latter, in individuals with normal P-tau, seemingly are not enough to cause symptoms on its own. Indeed, A $\beta 42$ levels have been shown to change in CSF years before the onset of actual symptoms [25, 45]. In our material, prodromal $\mathrm{AD}$ patients with pathological levels of $\mathrm{A} \beta 42$, but normal levels of P-tau, seem to need the added burden of white matter changes in order to develop cognitive difficulties.

Brain reserve and cognitive reserve refer to an individual's capacity for buffering brain pathology. It is a hypothetical concept proposed to account for the discrepancy between the degree of observed brain damage, or pathology, and its clinical manifestations [46, 47]. Clinicopathological studies have shown that many elderly people with quite extensive pathological AD-related lesions in the brain do not clinically manifest cognitive impairment, suggesting that structural and functional compensations could buffer the effects of neuropathology [48]. While the term 'brain reserve' is commonly used to describe structural factors, such as brain size and neuronal count, the term 'cognitive reserve' denotes more functional buffering factors, such as efficient cognitive processes and better compensatory processes [46]. The reserve can be viewed as the sum of the lifetime input of factors, some offering protection and some increasing vulnerability [49]. Many factors might erode this reserve. Each factor might not cause symptoms on its own, but together they push the individual over 
Hertze et al.: Tau Pathology and Parietal White Matter Lesions Have Independent but Synergistic Effects on Early Development of Alzheimer's Disease

the threshold. Our study shows that the addition of WMLs in the parietal regions might amplify the impact of AD pathology, advancing the onset of cognitive symptoms and shortening the time to dementia.

In conclusion, our findings support the hypothesis that AD is a syndrome, likely caused by many types of interacting pathologies. Based on our results, we suggest that WMLs in the parietal lobes reduce the reserve capacity of the brain, making it harder for an individual to compensate for other conditions targeting neurons, such as AD-related pathology. This might be valuable information for the clinician faced with the difficult task of foreseeing the future for patients with mild memory complaints and could provide insights into the causes of AD.

\section{References}

1 Comas-Herrera A, Wittenberg R, Pickard L, Knapp M, MRC-CFAS: Cognitive impairment in older people: its implications for future demand for services and costs. 2003. www.pssru.ac.uk/pdf/dp1728_2.pdf.

-2 Lowin A, Knapp M, McCrone P: Alzheimer's disease in the UK: comparative evidence on cost of illness and volume of health services research funding. Int J Geriatr Psychiatry 2001;16:1143-1148.

-3 Ferri CP, Prince M, Brayne C, Brodaty H, Fratiglioni L, Ganguli M, et al: Global prevalence of dementia: a Delphi consensus study. Lancet 2005;366:2112-2117.

4 Reed BR, Mungas D, Farias ST, Harvey D, Beckett L, Widaman K, et al: Measuring cognitive reserve based on the decomposition of episodic memory variance. Brain 2010;133:2196-2209.

-5 Sicras A, Rejas J, Arco S, Flores E, Ortega G, Esparcia A, et al: Prevalence, resource utilization and costs of vascular dementia compared to Alzheimer's dementia in a population setting. Dement Geriatr Cogn Disord 2005;19:305-315.

6 Blennow K, de Leon MJ, Zetterberg H: Alzheimer's disease. Lancet 2006;368:387-403.

7 Petersen RC: Mild cognitive impairment as a diagnostic entity. J Intern Med 2004;256:183-194.

-8 Straaten ECW, Harvey D, Scheltens P, Barkhof F, Petersen RC, Thal LJ, et al: Periventricular white matter hyperintensities increase the likelihood of progression from amnestic mild cognitive impairment to dementia. J Neurol 2008;255:1302-1308.

-9 Wolf H, Ecke GM, Bettin S, Dietrich J, Gertz HJ: Do white matter changes contribute to the subsequent development of dementia in patients with mild cognitive impairment? A longitudinal study. Int J Geriatr Psychiatry 2000;15:803-812.

10 Smith EE, Egorova S, Blacker D, Killiany RJ, Muzikansky A, Dickerson BC, et al: Magnetic resonance imaging white matter hyperintensities and brain volume in the prediction of mild cognitive impairment and dementia. Arch Neurol 2008;65:94-100.

11 Staekenborg SS, Koedam ELGE, Henneman WJP, Stokman P, Barkhof F, Scheltens P, et al: Progression of mild cognitive impairment to dementia: contribution of cerebrovascular disease compared with medial temporal lobe atrophy. Stroke 2009;40:1269-1274.

-12 Heyman A, Fillenbaum GG, Welsh-Bohmer KA, Gearing M, Mirra SS, Mohs RC, et al: Cerebral infarcts in patients with autopsy-proven Alzheimer's disease: CERAD, part XVIII. Consortium to Establish a Registry for Alzheimer's Disease. Neurology 1998;51:159-162.

13 Snowdon DA, Greiner LH, Mortimer JA, Riley KP, Greiner PA, Markesbery WR: Brain infarction and the clinical expression of Alzheimer disease. The Nun Study. JAMA 1997;277:813-817.

14 American Psychiatric Association: Diagnostic and Statistical Manual of Mental Disorders: DSM-III-R. Arlington, American Psychiatric Association, 1987.

15 McKhann G, Drachman D, Folstein M, Katzman R, Price D, Stadlan EM: Clinical diagnosis of Alzheimer's disease: report of the NINCDS-ADRDA Work Group under the auspices of Department of Health and Human Services Task Force on Alzheimer's Disease. Neurology 1984;34:939-944.

-16 Román GC, Tatemichi TK, Erkinjuntti T, Cummings JL, Masdeu JC, Garcia JH, et al: Vascular dementia: diagnostic criteria for research studies. Report of the NINDS-AIREN International Workshop. Neurology 1993;43: 250-260.

17 Erkinjuntti T, Inzitari D, Pantoni L, Wallin A, Scheltens P, Rockwood K, et al: Research criteria for subcortical vascular dementia in clinical trials. J Neural Transm Suppl 2000;59:23-30.

18 McKeith IG, Perry EK, Perry RH: Report of the second dementia with Lewy body international workshop: diagnosis and treatment. Consortium on Dementia with Lewy Bodies. Neurology 1999;53:902-905.

19 Blennow K, Hampel H, Weiner M, Zetterberg H: Cerebrospinal fluid and plasma biomarkers in Alzheimer disease. Nat Rev Neurol 2010;6:131-144.

20 Hertze J, Minthon L, Zetterberg H, Vanmechelen E, Blennow K, Hansson O: Evaluation of CSF biomarkers as predictors of Alzheimer's disease: a clinical follow-up study of 4.7 years. J Alzheimers Dis 2010;21:11191128. 
21 Wahlund LO, Barkhof F, Fazekas F, Bronge L, Augustin M, Sjögren M, et al: A new rating scale for age-related white matter changes applicable to MRI and CT. Stroke 2001;32:1318-1322.

22 Bronge L: Magnetic resonance imaging in dementia. A study of brain white matter changes. Acta Radiol Suppl 2002;1-32.

23 Altman D: Practical Statistics for Medical Research. London, Chapman Hall, 1991.

-24 Palmqvist S, Wattmo C, Bronge C, Zhang Y, Wahlund L-O, Nägga K: Association between subcortical lesions and behavioural and psychological symptoms in patients with Alzheimer's disease. Dement Geriatr Cogn Disord 2011;32:417-423.

-25 Bateman RJ, Xiong C, Benzinger TLS, Fagan AM, Goate A, Fox NC, et al: Clinical and biomarker changes in dominantly inherited Alzheimer's disease. N Engl J Med 2012;367:795-804.

26 Giannakopoulos P, Gold G, Kövari E, Gunten A, Imhof A, Bouras C, et al: Assessing the cognitive impact of Alzheimer disease pathology and vascular burden in the aging brain: the Geneva experience. Acta Neuropathol 2006;113:1-12.

-27 Drago V, Babiloni C, Bartrés-Faz D, Caroli A, Bosch B, Hensch T, et al: Disease tracking markers for Alzheimer's disease at the prodromal (MCI) stage. J Alzheimers Dis 2011;26(suppl 3):159-199.

-28 Bennett DA, Schneider JA, Wilson RS, Bienias JL, Arnold SE: Neurofibrillary tangles mediate the association of amyloid load with clinical Alzheimer disease and level of cognitive function. Arch Neurol 2004;61:378-384.

29 Gómez-Isla T, Hollister R, West H, Mui S, Growdon JH, Petersen RC, et al: Neuronal loss correlates with but exceeds neurofibrillary tangles in Alzheimer's disease. Ann Neurol 1997;41:17-24.

-30 Lace G, Savva GM, Forster G, de Silva R, Brayne C, Matthews FE, et al: Hippocampal tau pathology is related to neuroanatomical connections: an ageing population-based study. Brain 2009;132:1324-1334.

-31 Buchhave P, Minthon L, Zetterberg H, Wallin AK, Blennow K, Hansson O: Cerebrospinal fluid levels of $\beta$-amyloid 1-42, but not of tau, are fully changed already 5 to 10 years before the onset of Alzheimer dementia. Arch Gen Psychiatry 2012;69:98-106.

-32 Buerger K, Ewers M, Pirttilä T, Zinkowski R, Alafuzoff I, Teipel SJ, et al: CSF phosphorylated tau protein correlates with neocortical neurofibrillary pathology in Alzheimer's disease. Brain 2006;129:3035-3041.

-33 Hansson O, Zetterberg H, Buchhave P, Londos E, Blennow K, Minthon L: Association between CSF biomarkers and incipient Alzheimer's disease in patients with mild cognitive impairment: a follow-up study. Lancet Neurol 2006;5:228-234.

-34 Visser PJ, Verhey F, Knol DL, Scheltens P, Wahlund L-0, Freund-Levi Y, et al: Prevalence and prognostic value of CSF markers of Alzheimer's disease pathology in patients with subjective cognitive impairment or mild cognitive impairment in the DESCRIPA study: a prospective cohort study. Lancet Neurol 2009;8:619-627.

-35 Mattsson N, Zetterberg H, Hansson O, Andreasen N, Parnetti L, Jonsson M, et al: CSF biomarkers and incipient Alzheimer disease in patients with mild cognitive impairment. JAMA 2009;302:385-393.

-36 Shaw LM, Vanderstichele H, Knapik-Czajka M, Clark CM, Aisen PS, Petersen RC, et al: Cerebrospinal fluid biomarker signature in Alzheimer's disease neuroimaging initiative subjects. Ann Neurol 2009;65:403-413.

-37 Englund E: Neuropathology of white matter changes in Alzheimer's disease and vascular dementia. Dement Geriatr Cogn Disord 1998;9(suppl 1):6-12.

-38 Schneider JA, Arvanitakis Z, Bang W, Bennett DA: Mixed brain pathologies account for most dementia cases in community-dwelling older persons. Neurology 2007;69:2197-2204.

-39 Esiri MM, Nagy Z, Smith MZ, Barnetson L, Smith AD: Cerebrovascular disease and threshold for dementia in the early stages of Alzheimer's disease. Lancet 1999;354:919-920.

40 de Leeuw FE, Barkhof F, Scheltens P: Progression of cerebral white matter lesions in Alzheimer's disease: a new window for therapy? J Neurol Neurosurg Psychiatry 2005;76:1286-1288.

-41 Targosz-Gajniak M, Siuda J, Ochudło S, Opala G: Cerebral white matter lesions in patients with dementia - from MCI to severe Alzheimer's disease. J Neurol Sci 2009;283:79-82.

42 Maruyama M, Matsui T, Tanji H, Nemoto M, Tomita N, Ootsuki M, et al: Cerebrospinal fluid tau protein and periventricular white matter lesions in patients with mild cognitive impairment: implications for 2 major pathways. Arch Neurol 2004;61:716-720.

-43 Grambaite R, Stenset V: White matter diffusivity predicts memory in patients with subjective and mild cognitive impairment and normal CSF total tau levels. J Int Neuropsychol Soc 2010;16:58-69.

44 Gootjes L, Teipel S, Zebuhr Y, Schwarz R, Leinsinger G, Scheltens P, et al: Regional distribution of white matter hyperintensities in vascular dementia, Alzheimer's disease and healthy aging. Dement Geriatr Cogn Disord 2004;18:180-188.

45 Zetterberg H, Blennow K, Hanse E: Amyloid beta and APP as biomarkers for Alzheimer's disease. Exp Gerontol 2010;45:23-29.

46 Stern Y: Cognitive reserve. Neuropsychologia 2009;47:2015-2028.

-47 Stern Y: What is cognitive reserve? Theory and research application of the reserve concept. J Int Neuropsychol Soc 2002;8:448-460.

48 Fratiglioni L, Wang HX: Brain reserve hypothesis in dementia. J Alzheimers Dis 2007;12:11-22.

49 Richards M, Deary IJ: A life course approach to cognitive reserve: a model for cognitive aging and development? Ann Neurol 2005;58:617-622. 